EPJ Web of Conferences 107, 05002 (2016)

DOI: $10.1051 /$ epjconf/201610705002

(c) Owned by the authors, published by EDP Sciences, 2016

\title{
Impact of phonon coupling on the radiative nuclear reaction characteristics
}

\author{
Oleg Achakovskiy ${ }^{1, a}$, Alexander Avdeenkov ${ }^{1}$, and Sergei Kamerdzhiev² \\ ${ }^{1}$ Institute for Physics and Power Engineering, Obninsk, Russia \\ ${ }^{2}$ National Research Centre "Kurchatov Institute", Moscow, Russia
}

\begin{abstract}
The pygmy dipole resonance and photon strength functions (PSF) in stable and unstable Ni and Sn isotopes are calculated within the microscopic self-consistent version of the extended theory of finite Fermi systems in the quasiparticle time blocking approximation. The approach includes phonon coupling (PC) effects in addition to the standard QRPA approach. The Skyrme force SLy4 is used. A pygmy dipole resonance in ${ }^{72} \mathrm{Ni}$ is predicted at the mean energy of $12.4 \mathrm{MeV}$ exhausting $25.7 \%$ of the total energy-weighted sum rule. With our microscopic E1 PSFs in the EMPIRE 3.1 code, the following radiative nuclear reaction characteristics have been calculated for several stable and unstable even-even $\mathrm{Sn}$ and $\mathrm{Ni}$ isotopes: 1) neutron capture cross sections, 2) corresponding neutron capture gamma-spectra, 3) average radiative widths of neutron resonances. Here, three variants of the microscopic nuclear level density models have been used and a comparison with the phenomenological generalized superfluid model has been performed. In all the considered properties, including the recent experimental data for PSF in Sn isotopes, the PC contributions turned out to be significant, as compared with the QRPA one, and necessary to explain the available experimental data.
\end{abstract}

\section{Introduction}

The information about photon strength function (PSF) is necessary to calculate all characteristics of nuclear reactions with gamma-rays, in particular, the radiative neutron capture cross sections, which are of great astrophysical [1] and nuclear engineering [2] interest. Commonly, one parametrizes the PSF phenomenologically using, for example, generalized Lorentzian models [3, 4]. The usual definition of PSF contains transitions between excited states. For this reason, in order to calculate the PSF, the known Brink-Axel hypothesis is used which states that on each excited state it is possible to build a giant dipole resonance (at present, any giant resonance) including it's lowlying part. In this low-lying energy region, there exists the so-called Pygmy-Dipole Resonance (PDR). It exhausts typically about $1-2 \%$ of the Energy Weighted Sum Rule (EWSR) but, nevertheless, it can significantly increase the radiative neutron capture cross section and affect the nucleosynthesis of neutron-rich nuclei by the r-process [1]. In neutron-rich nuclei, for example, ${ }^{68} \mathrm{Ni}$ [5] and, probably, ${ }^{72} \mathrm{Ni},{ }^{74} \mathrm{Ni}$, the EWSR fraction is much larger. Note that for nuclei with small neutron separation energy, less than typically 3-4 MeV, the PDR properties are changed significantly [1], and therefore, phenomenological systematics obtained by fitting characteristics of stable nuclei cannot be applied. Because the Brink-Axel hypothesis, probably, is valid, the PSF is connected very simply with the photoabsorption cross section and, therefore, with the PDR field, see $[3,6,7]$. For all these reasons, during the last

\footnotetext{
ae-mail: oachakovskiy@ippe.ru
}

decade there has been an increasing interest in the investigations of the excitations in the PDR energy region manifested both in "pure" low-energy nuclear physics $[6,8]$ and in the nuclear data field $[1,3,4]$.

The experiments in the PDR energy region [9-12] have given additional information about the PDR and PSF structures. The PSF structures at $8-9 \mathrm{MeV}$ in six Sn isotopes obtained by the Oslo method $[9,10]$ could not be explained within the standard phenomenological approach. In order to explain the experiment, it was necessary to add "by hand" some additional strength of about $1-2 \%$ of the EWSR.

Given the importance of PSF both in astrophysics [1] and nuclear engineering [2], microscopic investigations are required, especially when extrapolations to exotic nuclei are needed. Mean-field approaches using effective nucleon interactions, such as the Hartree-Fock-Bogoliubov method and the quasi-particle random-phase approximation (HFB+QRPA) [1], allow systematic self-consistent studies of isotope chains, and indeed have been included in modern nuclear reaction codes like EMPIRE [13] and TALYS [14]. Such an approach is of higher predictive power in comparison with phenomenological models. However, as we discuss below and as confirmed by recent experiments, the HFB+QRPA approach is necessary but not sufficient. To be exact, it should be complemented by the effect describing the interaction of single-particle degrees of freedom with the low-lying collective phonon degrees of freedom, known as the phonon coupling (PC).

The results [12] directly confirm the necessity to go beyond the HFB+QRPA method because the PSF struc-

This is an Open Access article distributed under the terms of the Creative Commons Attribution License 4.0, which permits unrestricted use, distribution, and reproduction in any medium, provided the original work is properly cited. 
tures observed in [10] could not be explained within the HFB+QRPA approach. In particular, the PC effects discussed in Refs. [7, 15, 16] may be at the origin of such an extra strength. Note that the microscopic PSFs which contained transitions between the ground and excited states, i.e. the PSFs values at the energy point near the neutron separation energy, have already been estimated long ago within the quasiparticle-phonon model approach, which also includes the phonon coupling [17].

In this work, we use the self-consistent version of the extended theory of finite Fermi systems (ETFFS) [15] in the quasi-particle time blocking approximation (QTBA) [18]. Our ETFFS (QTBA) method, or simply QTBA, includes self-consistently the QRPA and $\mathrm{PC}$ effects and the single-particle continuum in a discrete form. Details of the method are described in Ref. [16]. The method allows us to investigate the impact of the PC on nuclear reaction in both stable and unstable nuclei. We calculate the microscopic PSFs in several $\mathrm{Sn}$ and $\mathrm{Ni}$ isotopes and use them in the EMPIRE code to estimate the neutron capture cross sections, corresponding capture gamma-ray spectra and average radiative widths.

\section{PSFs}

To calculate the strength function $S(\omega)=d B(E 1) / d E$ $[15,16]$, which is connected with the PSF $f(E 1)$ as $f(E 1, \omega)\left[\mathrm{MeV}^{-3}\right]=3.487 \cdot 10^{-7} S(\omega)\left[\mathrm{fm}^{2} \mathrm{MeV}^{-1}\right]$, we use the well-known SLy4 Skyrme force [19]. The ground state is calculated within the HFB method using the spherical code HFBRAD [20]. The residual interaction for the (Q)RPA and QTBA calculations is derived as the second derivative of the Skyrme functional. In all our calculations we use a smoothing parameter of $200 \mathrm{keV}$ which effectively accounts for correlations beyond the considered PC which do not show a strong energy dependence. Such a choice guarantees the correct description of all three characteristics of giant resonances, including their widths [15] and, what is important here, this value approximately coincides with the experimental resolution of the Oslo method [10].

Figures 1, 2 show the calculations for three variants of the radiative strength function for the ${ }^{116,118,122} \mathrm{Sn}$ isotopes: the phenomenological enhanced generalized Lorentzian (EGLO) [3], as well as the microscopic QRPA and QTBA in each nucleus, in comparison to the existing experimental data for these nuclei.

We obtained:

(i) In contrast to phenomenological approaches, all nuclei exhibit structures caused by the effects of the QRPA and the effects of phonon coupling. In this case, the difference of the QTBA from the QRPA, i.e., the contribution from phonon coupling, becomes noticeable at energies $E<10 \mathrm{MeV}$. More precisely, structures at about $E>5$ $\mathrm{MeV}$ are due only to the effect of phonon coupling. This corresponds to the mentioned experimental data $[9,10]$.

(ii) As expected, the EGLO curves are in general agreement with experimental data for energies above the neutron threshold, because the EGLO phenomenology

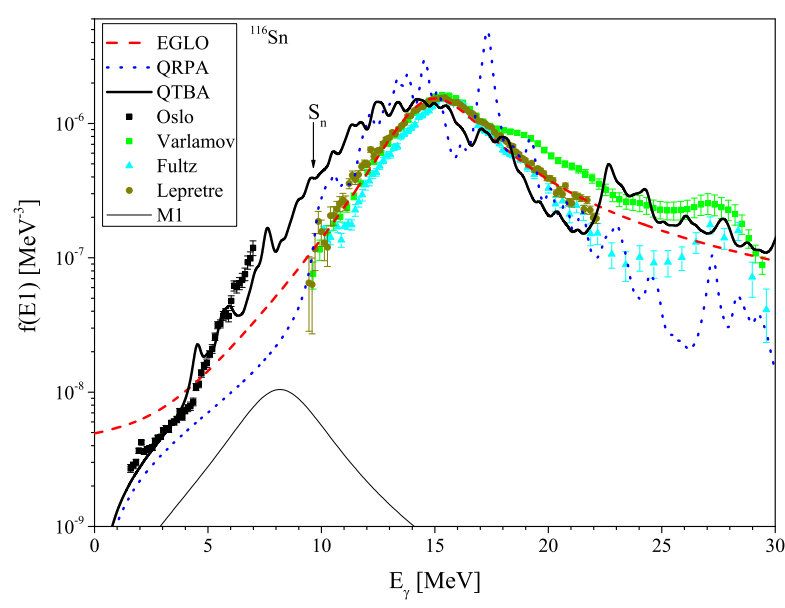

Figure 1. (Color online) The $E 1$ photon strength functions for ${ }^{116} \mathrm{Sn}$. The dashed lines are obtained within the phenomenological variant of the EGLO [3], the dotted line is the QRPA calculation, and the solid line is the QTBA calculation (the complete microscopic calculation). The arrow marks the neutron separation energy. The experimental data are taken from [9, 21-23].

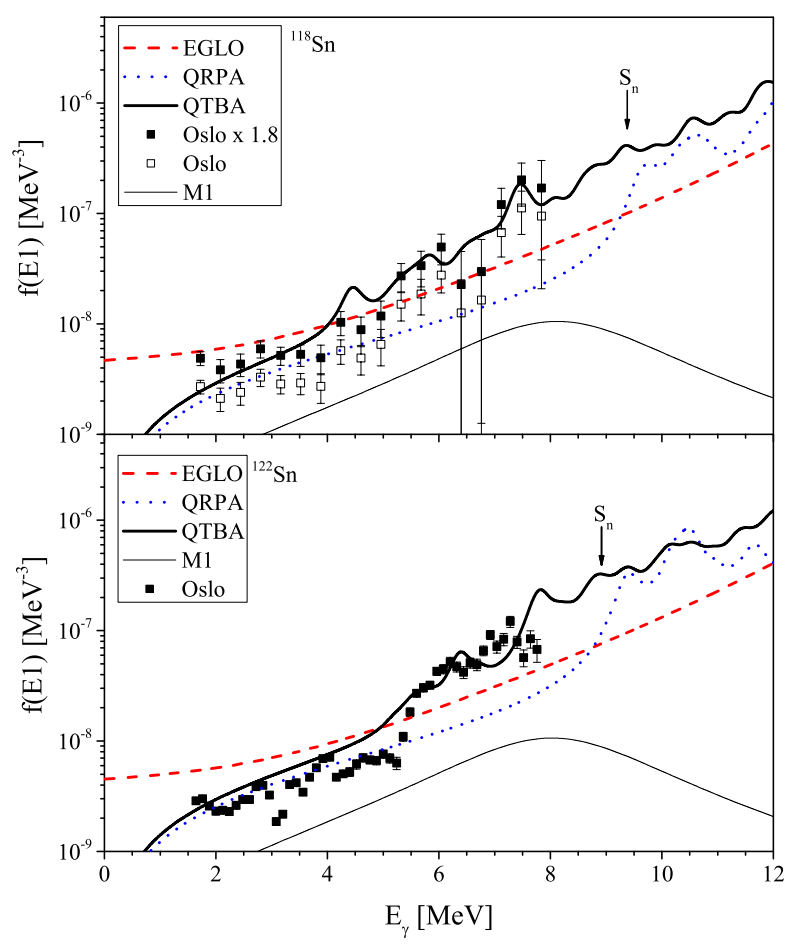

Figure 2. (Color online) The same as for Fig. 1, but for ${ }^{118} \mathrm{Sn}$ and ${ }^{122} \mathrm{Sn}$ and for the PDR energy region. The experimental data are taken from $[9,10]$.

was chosen from the corresponding experiments. As it can be seen in Fig. 1 for ${ }^{116} \mathrm{Sn}$, our calculations in the giant dipole resonance (GDR) energy range are in worse agreement with the experiment than those in the energy range up to the binding energy. One of the main reasons for this is that the smoothing parameter for energies above the nucleon separation energy should depend on the energy. However, as was shown in [24], all observed integral 


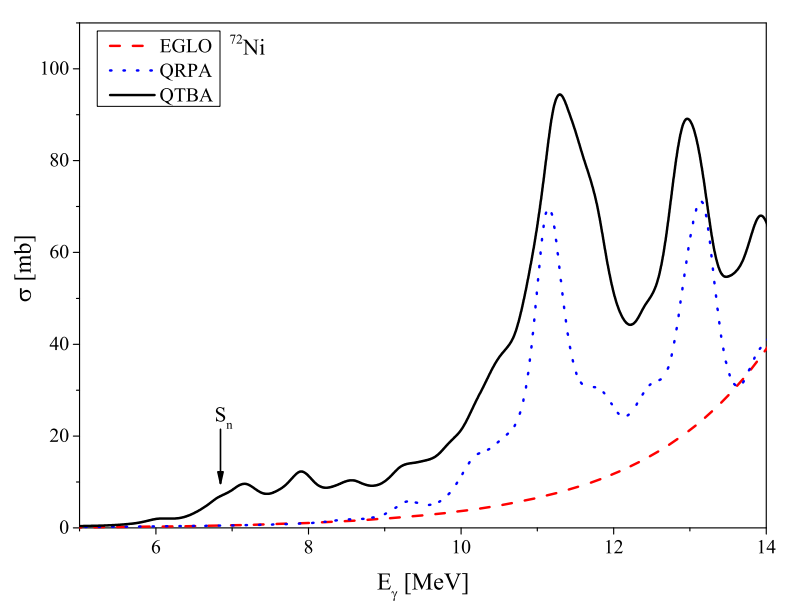

Figure 3. (Color online) The calculated photoabsorption cross section in ${ }^{72} \mathrm{Ni}$. See text for details.

characteristics, including the most important of them - the width of the GDR - are described satisfactorily with a parameter of $200 \mathrm{keV}$ used here.

In Ref. [5], the PDR in the unstable ${ }^{68} \mathrm{Ni}$ nucleus was measured. It was found that the PDR is in the range of 7-13 MeV, has a maximum at an energy of $11 \mathrm{MeV}$, and exhausts about $5 \%$ of the EWSR. The neutron separation energy is $7.8 \mathrm{MeV}$, i.e. the PDR in this nucleus is located noticeably above the neutron emission threshold. The situation in the ${ }^{72} \mathrm{Ni}$ nucleus should be similar. Predictions for this nucleus are of interest because of the possibility of the corresponding experiments. The results of the calculations for the PDR in three nickel isotopes are presented in Table 1 in comparison to the results for the stable ${ }^{58} \mathrm{Ni}$ nucleus and shown in Fig. 3 for the PDR in ${ }^{72} \mathrm{Ni}$.

In Table 2, the integral parameters of the PDR are given for two our PSF models, i.e. microscopic QRPA and QTBA (QRPA+PC). To compare, the $6 \mathrm{MeV}$ interval, where the PDR was observed in ${ }^{68} \mathrm{Ni}$, is considered. In this interval, the PDR characteristics have been approximated, as usual, using a Lorentz curve by fitting the three moments of the Lorentzian and theoretical curves [15]. A reasonable agreement with experimental data [5] for ${ }^{68} \mathrm{Ni}$ is obtained. Earlier, a similar calculation was performed for ${ }^{68} \mathrm{Ni}$ [25] using the relativistic QTBA, with two phonon contributions additionally taken into account. Concomitantly, the PDR characteristics in ${ }^{72} \mathrm{Ni}$ have been estimated leading in this interval to a mean energy of $12.4 \mathrm{MeV}$ and the large strength of $25.7 \%$ of the total EWSR. In all three isotopes, a large PC contribution to the PDR strength has been found.

\section{Neutron radiative capture cross sections}

As an example, the neutron radiative capture cross sections for ${ }^{119} \mathrm{Sn}$ obtained with the QRPA and QRPA+PC photon E1 strength functions are shown in Fig. 4. We also show the uncertainty bands obtained when considering various nuclear level density (NLD) models [26-28].
Table 1. Integral characteristics of the PDR (mean energy $E$ in $\mathrm{MeV}$ and fraction of the EWSR) calculated for ${ }^{58} \mathrm{Ni},{ }^{72} \mathrm{Ni}$ (interval 8-14 MeV) and for ${ }^{68} \mathrm{Ni}$ (interval 7-13 MeV) (see text for details).

\begin{tabular}{lllllll}
\hline \hline \multirow{2}{*}{ Nuclei } & \multicolumn{2}{l}{ QRPA } & \multicolumn{3}{l}{ QTBA } & \multicolumn{2}{l}{ Exp. } \\
\cline { 2 - 7 } & $E$ & $\%$ & $E$ & $\%$ & $E$ & $\%$ \\
\hline${ }^{58} \mathrm{Ni}$ & 13.3 & 6.0 & 14.0 & 11.7 & & \\
${ }^{68} \mathrm{Ni}$ & 11.0 & 4.9 & 10.8 & 8.7 & $11[5]$ & $\approx 5[5]$ \\
${ }^{72} \mathrm{Ni}$ & 12.4 & 14.7 & 12.4 & 25.7 & & \\
\hline \hline
\end{tabular}

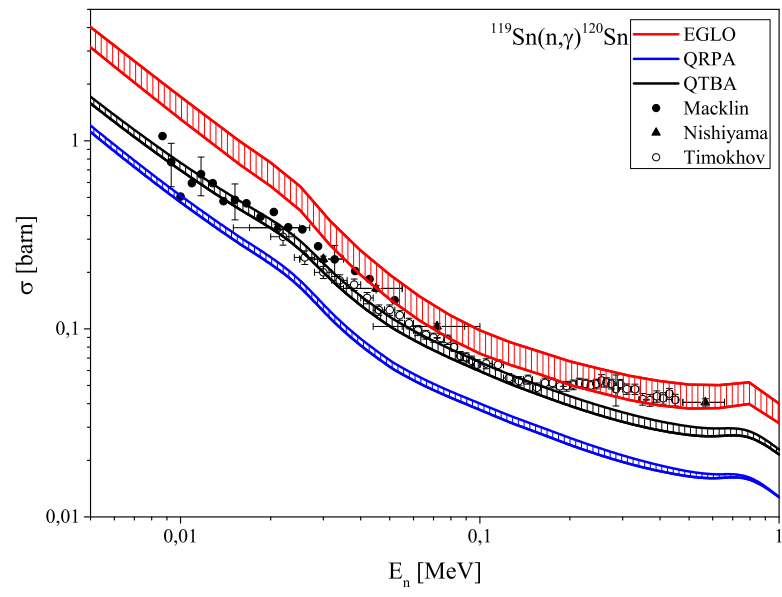

Figure 4. (Color online) ${ }^{119} \mathrm{Sn}(\mathrm{n}, \gamma)$ cross section calculated with the EGLO (red), QRPA (blue) and QTBA (black) PSF. The uncertainty bands depict the uncertainties affecting the nuclear level density predictions [26-28]. Experimental data are taken from Refs. [29-31].

One can clearly see that the agreement with experiment up to $0.2 \mathrm{MeV}$ is only possible when the PC is taken into account. The use of the EGLO PSF with the microscopic NLD model gives an agreement with experiment at higher neutron energies. The details see also in [32].

\section{Capture gamma-ray spectra}

The corresponding capture gamma-ray spectra calculated for the neutron energies of $52 \mathrm{keV}$ and $570 \mathrm{keV}$ are displayed in Fig. 5 and compared with with the experimental data [30] and the results obtained with microscopic and phenomenological EGLO PSF models. Here, the microscopic HFB+combinatorial NLD model [26] of nuclear level densities is adopted. As compared with the phenomenological GSM NLD model, which was used in [33], the agreement with experiment is better. Our results show that the PC contribution is significant. For all three PSF variants some structures have been found.

We have performed the same calculations for the unstable ${ }^{68} \mathrm{Ni}$ for the neutron energy $100 \mathrm{keV}$ (see Fig. 6). One can see a large difference between results with our two microscopic and the phenomenological EGLO PSF models which confirms the necessity of using the microscopic approach for unstable nuclei. The similar situation is for the GSM NLD model in Ref. [33]. 


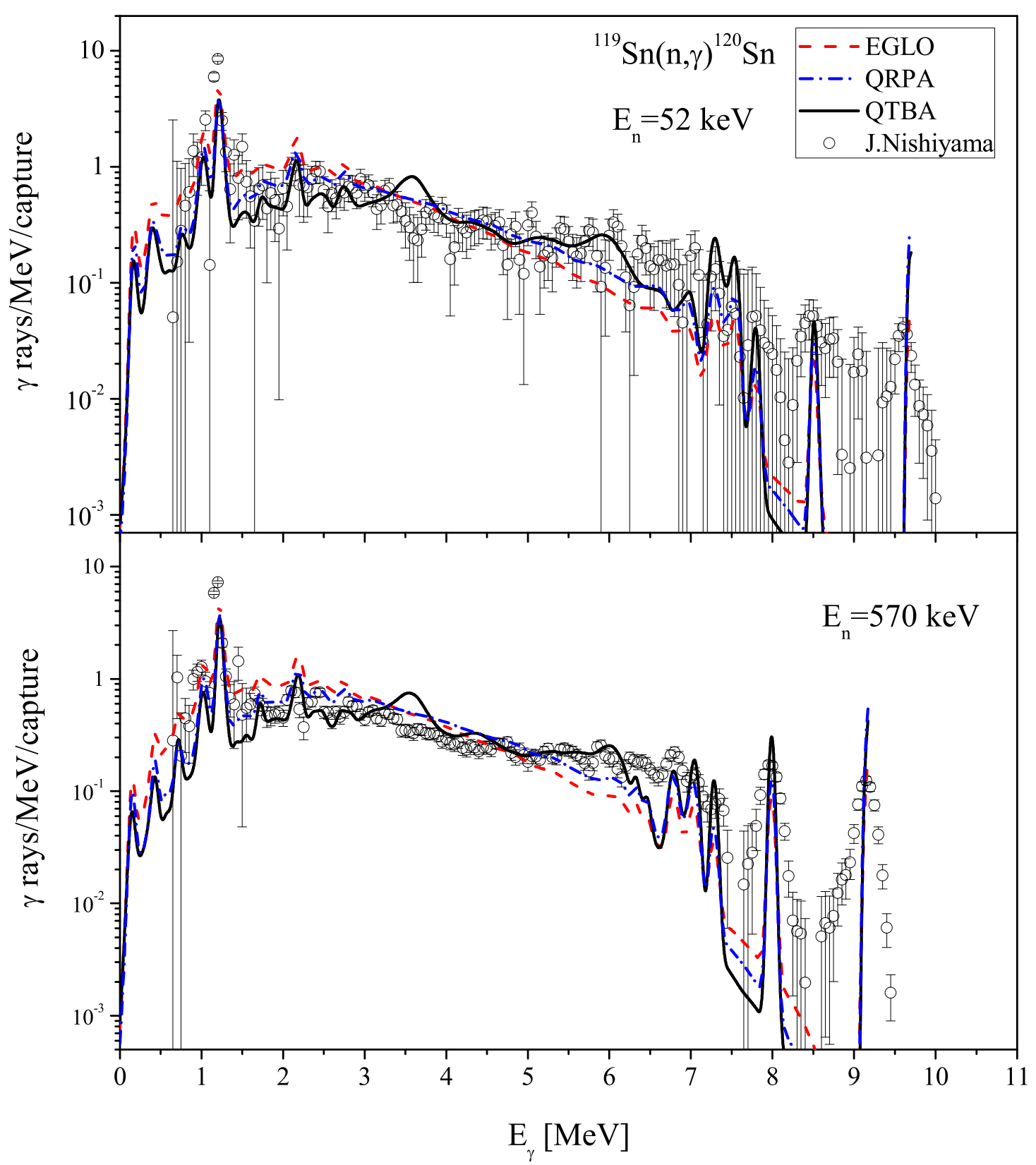

Figure 5. (Color online) Gamma-ray spectra from ${ }^{119} \mathrm{Sn}(\mathrm{n}, \gamma)$ for the neutron energy of $52 \mathrm{keV}$ and $570 \mathrm{keV}$. The microscopic HFB+combinatorial NLD model [26] has been used. Experimental data was taken from [30].

\section{Average radiative widths}

Average radiative widths of neutron resonances $\Gamma_{\gamma}$ are very important properties of gamma-decay from high-energy nuclear states; they are used for calculations of radiative capture cross sections and other reactions with gammarays. There are a lot of experimental data for $\Gamma_{\gamma}[2,34]$. For $13 \mathrm{Sn}$ and Ni isotopes, we have calculated with EMPIRE 3.1 the values $\Gamma_{\gamma}$ with the EGLO and our QRPA and QTBA PSF models using the Generalised Superfluid Model (GSM) NLD [3] and the microscopic HFB plus combinatorial model [26]. The predictions are compared in Table 2 with experimental data [2], whenever available, and systematics [34]. We have found that the PC in stable nuclei increases the QRPA contribution in the direction of the systematics and, except for ${ }^{122} \mathrm{Sn}$ and ${ }^{124} \mathrm{Sn}$, where the increase is limited, the PC leads to an enhancement of about 50 to $200 \%$.

Our $\Gamma_{\gamma}$ results for ${ }^{118} \mathrm{Sn},{ }^{120} \mathrm{Sn},{ }^{60} \mathrm{Ni}$, and ${ }^{62} \mathrm{Ni}$ where experimental data (not systematics) exists, are of special interest. On the basis of the QTBA strength and the microscopic HFB plus combinatorial NLD [26], we obtain a good agreement with experiment for ${ }^{60} \mathrm{Ni},{ }^{62} \mathrm{Ni}$, and reasonable for ${ }^{118} \mathrm{Sn}$ and ${ }^{120} \mathrm{Sn}$. Note that on top of the $E 1$ strength, an $M 1$ contribution following the recommendation of Ref. [4] is included in the calculation of $\Gamma_{\gamma}$. The $M 1$ resonance contribution to $\Gamma_{\gamma}$ has been estimated using the GSM NLD model and the standard Lorentzian parametrization [4] with a width $\Gamma=4 \mathrm{MeV}$ 
Table 2. Average radiative widths $\Gamma_{\gamma}(\mathrm{meV})$ for s-wave neutrons. For each approach (EGLO, QRPA and QTBA) two NLD models are considered: the phenomenological GSM [3] (first lines) and the microscopic HFB plus combinatorial model [26] (second lines). See text for details.

\begin{tabular}{|c|c|c|c|c|c|c|c|c|c|c|c|c|c|}
\hline & ${ }^{110} \mathrm{Sn}$ & ${ }^{112} \mathrm{Sn}$ & ${ }^{116} \mathrm{Sn}$ & ${ }^{118} \mathrm{Sn}$ & ${ }^{120} \mathrm{Sn}$ & ${ }^{122} \mathrm{Sn}$ & ${ }^{124} \mathrm{Sn}$ & ${ }^{136} \mathrm{Sn}$ & ${ }^{58} \mathrm{Ni}$ & ${ }^{60} \mathrm{Ni}$ & ${ }^{62} \mathrm{Ni}$ & ${ }^{68} \mathrm{Ni}$ & ${ }^{72} \mathrm{Ni}$ \\
\hline \multirow{2}{*}{ EGLO } & 147.4 & 105.5 & 72.9 & 46.6 & 55.0 & 56.6 & 49.9 & 11.1 & 1096 & 474 & 794 & 166 & 134 \\
\hline & 207.9 & 160.3 & 108.9 & 106.7 & 124.3 & 110.2 & 128.7 & 295.0 & 2017 & 1882 & 1841 & 982.2 & 86.4 \\
\hline \multirow{2}{*}{ QRPA } & 45.6 & 34.4 & 30.4 & 22.1 & 23.8 & 27.9 & 22.3 & 11.2 & 358 & 594 & 623 & 75.4 & 83.8 \\
\hline & 71.0 & 49.7 & 44.3 & 40.3 & 43.0 & 50.1 & 68.9 & 447.8 & 450.8 & 1646 & 490.9 & 406.4 & 46.7 \\
\hline \multirow{2}{*}{ QTBA } & 93.5 & 65.7 & 46.8 & 33.1 & 34.1 & 35.8 & 27.9 & 12.3 & 1141 & 971 & 1370 & 392 & 154 \\
\hline & 119.9 & 87.0 & 58.4 & 58.1 & 61.5 & 64.0 & 84.8 & 509.2 & 1264 & 2800 & 2117 & 2330 & 53.8 \\
\hline Exp. ${ }_{[3]}^{[2]}$ & & & & $\begin{array}{l}117(20) \\
80(20)\end{array}$ & $100(16)$ & & & & & $2200(700)$ & $\begin{array}{l}2000(300) \\
2200(700)\end{array}$ & & \\
\hline \multirow{2}{*}{$M 1$} & 13.0 & 9.6 & 8.9 & 6.1 & 6.6 & 7.3 & 4.9 & 1.3 & 46.1 & 32 & 23.2 & 36.0 & 49.6 \\
\hline & 29.1 & 18.1 & 18.5 & 13.2 & 13.4 & 13.1 & 15.5 & 87.2 & 17.0 & 52 & 31.8 & 81.6 & 27.5 \\
\hline System. & 112 & 109 & 107 & 106 & 105 & 104 & 103 & 73 & 2650 & 1900 & 1300 & 420 & 320 \\
\hline
\end{tabular}

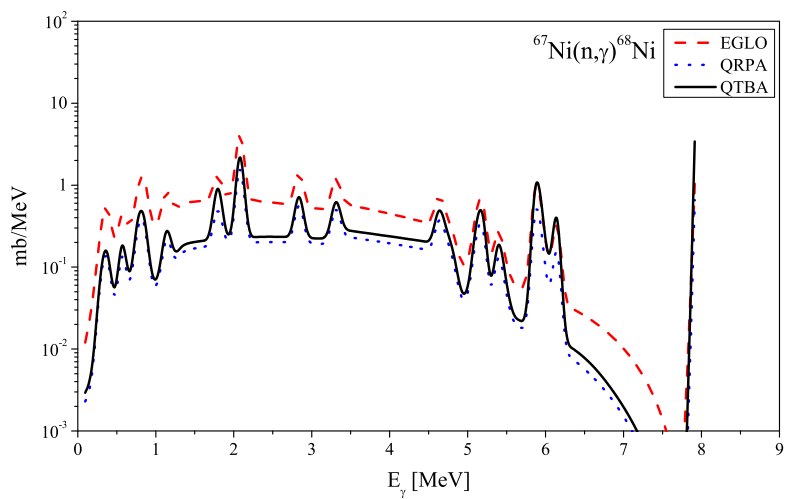

Figure 6. (Color online) Gamma-ray spectra from ${ }^{68} \mathrm{Ni}(\mathrm{n}, \gamma)$ for the neutron energy of $100 \mathrm{keV}$. The microscopic HFB+combinatorial NLD model [26] has been used.

(note that such a large $\Gamma$ value is questionable, as discussed in Ref. [35]). Such a contribution is found to be of the order of (10-12)\% of the values in the first line of Table 2 for Sn isotopes and $4 \%, 3 \%, 22 \%$, and $16 \%$ for ${ }^{58} \mathrm{Ni},{ }^{62} \mathrm{Ni},{ }^{68} \mathrm{Ni}$, and ${ }^{72} \mathrm{Ni}$, respectively. The agreement of the $\Gamma_{\gamma}$ values with experiment is found to deteriorate if use is made of the EGLO or QRPA strengths, but also of the GSM NLD. One can also see that for stable nuclei, the combinatorial NLD model results are in a better agreement with the systematics [3] than those obtained with the GSM model. As far as the EGLO model is concerned, we see that similar conclusions can be drawn.

\section{Conclusion}

The characteristics of nuclear reactions with gammarays have been calculated within the microscopic selfconsistent approach which takes into account the QRPA and PC effects and uses the SLy4 Skyrme force. Such a self-consistent approach is of particular relevance for nuclear astrophysics. A reasonable agreement with available experimental data has been obtained thanks to PC. We predict the PDR in the spherical ${ }^{72} \mathrm{Ni}$ nucleus at $12.4 \mathrm{MeV}$ with a very large strength corresponding to $25.7 \%$ of the
EWSR. For the first time, the average radiative widths have been calculated microscopically with the PC taken into account. In all the considered quantities, the contribution of PC turned out to be significant. These results confirm the necessity of including the PC effects into the theory of nuclear data both for stable and unstable nuclei. The phenomenological GSM NLD model used in EMPIRE 3.1 gives, on the whole, the worse results than the microscopic HFB plus combinatorial NLD model.

The authors (O.A. and S.K.) are very thankful to Organizing Committee of the NSRT15 conference for the financial support of their participation in the conference.

\section{References}

[1] S. Goriely, E. Khan, V. Samyn, Nucl. Phys. A 739, 331 (2004)

[2] S.F. Mughabghab, Atlas of neutron Resonances, Resonance Parameters and Thermal Cross Sections $Z=1-100$ (Elsevier, Amsterdam, 2006)

[3] T. Belgya, O. Bersillon, R. Capote et al., Handbook for Calculations of Nuclear Reaction Data, RIPL2, IAEA-TECDOC-1506 (IAEA, Vienna, 2006) [http://www-nds.iaea.org/RIPL-2/]

[4] R. Capote, M. Herman, P. Oblozinsky et al., Nucl. Data Sheets 110, 3107 (2009); see also https://wwwnds.iaea.org/RIPL-3.

[5] O. Wieland, A. Bracco, F. Camera et al., Phys. Rev. Lett. 102, 092502 (2009)

[6] D. Savran, T. Aumann, A. Zilges, Prog. Part. Nucl. Phys. 70, 210 (2013)

[7] S.P. Kamerdzhiev, A.V. Avdeenkov, O.I. Achakovskiy, Phys. At. Nucl. 77, 1303 (2014)

[8] N. Paar, D. Vretenar, E. Khan, G. Colo, Rep. Prog. Phys. 70, 691 (2007)

[9] H.K. Toft, A.C. Larsen, U. Agvaanluvsan et al., Phys. Rev. C 81, 064311 (2010)

[10] H.K. Toft, A. C. Larsen, A. Bürger et al., Phys. Rev. C 83, 044320 (2011)

[11] R. Schwengner, R. Massarczyk, G. Rusev et al., Phys. Rev. C 87, 024306 (2013) 
[12] H. Utsunomiya, S. Goriely, M. Kamata et al., Phys. Rev. C 84, 055805 (2011)

[13] M. Herman, R. Capote, B.V. Carlson et al., Nucl. Data Sheets, 108 (2007) 2655-2715; see also http://www.nndc.bnl.gov/empire/index.html.

[14] A.J. Koning and D. Rochman, Nucl. Data Sheets 113, 2841 (2012)

[15] S.Kamerdzhiev, J. Speth, G. Tertychny, Phys. Rep. 393, 1 (2004)

[16] A. Avdeenkov, S. Goriely, S. Kamerdzhiev, S. Krewald, Phys. Rev. C 83, 064316 (2011)

[17] V.G. Soloviev, Ch. Stoyanov and V.V. Voronov, Nucl. Phys. A 304, 503 (1978)

[18] V. Tselyaev, Phys. Rev. C 75, 024306 (2007)

[19] E. Chabanat, P. Bonche, P. Haensel, Nucl. Phys. A 635, 231 (1998)

[20] K.Bennaceur and J. Dobaczewski, Comp. Phys. Comm 168, 96 (2005)

[21] V.V. Varlamov, N.N. Peskov, D.S. Rudenko, and M.E. Stepanov, Vopr. At. Nauki Tekh., Ser. Yad. Konstanty 1, 2 (2003)

[22] S.C. Fultz, B.L. Berman, J.T. Coldwell et al., Phys. Rev. 186, 1255 (1969)

[23] A. Leprêtre, H. Beil, R. Bergere et al., Nucl. Phys. A 219, 39 (1974)

[24] O.I. Achakovskiy, A.V. Avdeenkov, and S.P. Kamerdzhiev, in Proceedings of the Int. Seminar on Interac- tion of Neutrons with Nuclei, ISINN22 (Dubna, May 27-30, 2014), p. 213.

[25] E. Litvinova, P. Ring, V. Tselyaev, Phys. Rev. Lett. 105, 022502 (2010)

[26] S. Goriely, S. Hilaire, A.J. Koning, Phys. Rev. C 78, 064307 (2008)

[27] A.J. Koning, S. Hilaire, S. Goriely, Nucl. Phys. A 810, 13 (2008)

[28] S. Hilaire, M. Girod, S. Goriely, and A.J. Koning, Phys. Rev. C 86, 064317 (2012)

[29] R.L. Macklin, T. Inada, J.H. Gibbons, Washington AEC Office Reports, No.1041, p.30 (1962)

[30] J. Nishiyama, M. Igashira, T. Ohsaki et al., J. Nucl. Sci. Technol. (Tokyo) 45, 352 (2008)

[31] V.M. Timokhov et al., Fiz.-Energ Institut, Obninsk Reports No.1921 (1988)

[32] O. Achakovskiy, A. Avdeenkov, S. Goriely et al., Phys. Rev. C 91, 034620 (2015)

[33] O.I. Achakovskiy, A.V. Avdeenkov, S.P. Kamerdzhiev and D.A. Voitenkov, in Proceedings of the Int. Seminar on Interaction of Neutrons with Nuclei, ISINN22 (Dubna, May 27-30, 2014), p. 207.

[34] Reference Input Parameter Library, RIPL-1, IAEATECDOC-1034 (1998)

[35] S.P. Kamerdzhiev, S.F. Kovalev, Phys. At. Nucl. 69, 418 (2006) 